\title{
PUMA-mediated intestinal epithelial apoptosis contributes to ulcerative colitis in humans and mice
}

\author{
Wei Qiu, ${ }^{1,2}$ Bin Wu, ${ }^{1,3}$ Xinwei Wang, ${ }^{1,2}$ Monica E. Buchanan,1,3 Miguel D. Regueiro, ${ }^{4}$ \\ Douglas J. Hartman, ${ }^{2}$ Robert E. Schoen, 1,4 Jian Yu,, 1,2 and Lin Zhang1,3 \\ 1University of Pittsburgh Cancer Institute, ${ }^{2}$ Department of Pathology, ${ }^{3}$ Department of Pharmacology and Chemical Biology, \\ and ${ }^{4}$ Department of Medicine, University of Pittsburgh School of Medicine, Pittsburgh, Pennsylvania, USA.
}

\begin{abstract}
Intestinal epithelial cell (IEC) apoptosis contributes to the development of ulcerative colitis (UC), an inflammatory bowel disease (IBD) that affects the colon and rectum. Therapies that target the inflammatory cytokine TNF have been found to inhibit IEC apoptosis in patients with IBD, although the mechanism of IEC apoptosis remains unclear. We therefore investigated the role of p53-upregulated modulator of apoptosis (PUMA), a p53 target and proapoptotic $\mathrm{BH3}$-only protein, in colitis and IEC apoptosis, using patient samples and mouse models of UC. In UC patient samples, PUMA expression was elevated in colitis tissues relative to that in uninvolved tissues, and the degree of elevation of PUMA expression correlated with the severity of colitis and the degree of apoptosis induction. In mice, PUMA was markedly induced in colonic epithelial cells following induction of colitis by either dextran sulfate sodium salt (DSS) or 2,4,6-trinitrobenzene sulfonic acid (TNBS). The induction of PUMA was p53-independent but required NF- $\kappa B$. Absence of PUMA, but neither absence of p53 nor that of another BH3-only protein (Bid), relieved DSS- and TNBS-induced colitis and inhibited IEC apoptosis. Furthermore, treating mice with infliximab (Remicade), a clinically used TNF-specific antibody, suppressed DSS- and TNBS-induced PUMA expression and colitis. These results indicate that PUMA induction contributes to the pathogenesis of colitis by promoting IEC apoptosis and suggest that PUMA inhibition may be an effective strategy to promote mucosal healing in patients with UC.
\end{abstract}

\section{Introduction}

The inflammatory bowel diseases (IBDs) ulcerative colitis (UC) and Crohn disease (CD) are thought to result from aberrant activation of the intestinal mucosal immune system (1). Although the pathogenesis of IBD remains unclear, a number of studies have suggested a role of abnormal apoptosis in the intestinal epithelial cells (IECs), resulting from increased cytokine production, such as TNF, IL, and interferon family members (2). Increased IEC apoptosis has been detected at the acute inflammatory sites in $\mathrm{UC}(3,4)$ and CD patients $(5)$. Induction of IEC apoptosis has also been described in a number of studies using murine colitis models (6-8). IEC apoptosis can disrupt intestinal mucosal integrity and barrier function and lead to other changes associated with colitis $(9,10)$. Furthermore, anti-TNF therapies for treating IBD patients were found to inhibit IEC apoptosis $(11,12)$. However, the molecular basis of IEC apoptosis in response to intestinal inflammation remains poorly understood.

p53-upregulated modulator of apoptosis (PUMA), a BH3-only Bcl-2 family member, was identified as a downstream target of the tumor suppressor p53 and a potent inducer of apoptosis in diverse tissues and cell types (13). The expression of PUMA is elevated in response to different stimuli through p53-dependent or -independent transcription (14). PUMA binds to antiapoptotic Bcl-2 family members to activate the proapoptotic members Bax and Bak to trigger mitochondrial dysfunction. This results in the release of

Authorship note: Wei Qiu and Bin Wu contributed equally to this work. Conflict of interest: The authors have declared that no conflict of interest exists. Citation for this article: J Clin Invest. 2011;121(5):1722-1732. doi:10.1172/JCI42917. several apoptogenic mitochondrial proteins, such as cytochrome $c$ and SMAC, leading to caspase activation and cell death $(15,16)$. Knockout of PUMA in human colon cancer cells or in mice leads to resistance to apoptosis induced by a number of stimuli, such as DNA damage, kinase inhibition, oncogene activation, and endoplasmic reticulum stress (13).

An emerging role of PUMA in gastrointestinal inflammation and tissue injury has been revealed by several recent studies. PUMA is directly activated by NF- $\mathrm{B}$, a key player in intestinal inflammation (17), in response to TNF- $\alpha$ treatment (18). TNF- $\alpha$-induced apoptosis in several tissues, including small intestine and colon, is significantly reduced in PUMA-deficient (PUMA-KO) mice (18). Lack of PUMA significantly compromised IEC apoptosis and intestinal damage induced by $\gamma$-irradiation (19). Using an acute ischemia/reperfusion model, we previously demonstrated that PUMA is necessary for IEC apoptosis and tissue injury induced by intestinal ischemia/reperfusion, which induces an inflammatory response (20). Furthermore, PUMA was found to be an important regulator of apoptosis in the immune system, and its expression is subject to regulation by immune modulators, such as glucocorticoids and growth hormones, and by bacterial or viral infection (13).

We hypothesized that PUMA is involved in the pathogenesis of colitis by mediating IEC apoptosis induced by intestinal inflammation. We found that PUMA is induced in patients with UC and mouse experimental colitis models, and its induction is correlated with severity of colitis. Our findings suggest that PUMA induction by TNF through NF- $\kappa \mathrm{B}$ contributes to colitis and IEC apoptosis, whereas its suppression by anti-TNFs attenuates colitis and IEC apoptosis. 
A

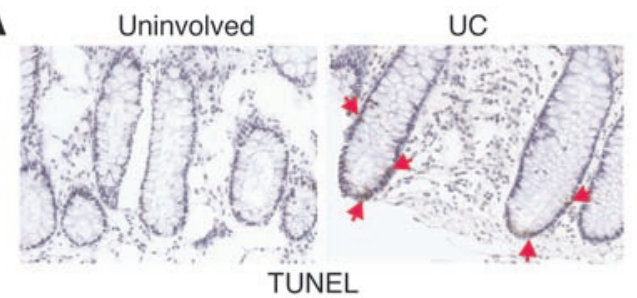

B

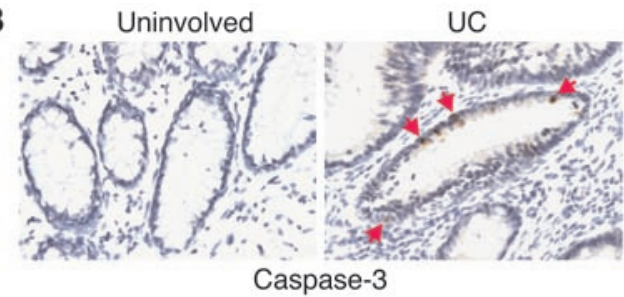

C

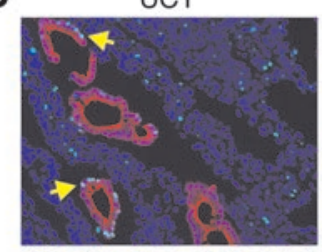

TUNEL/Cytokeratin/DAPI

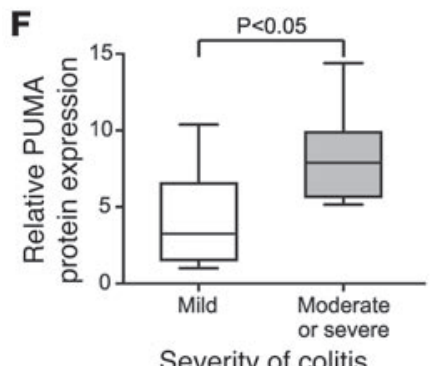

Severity of colitis
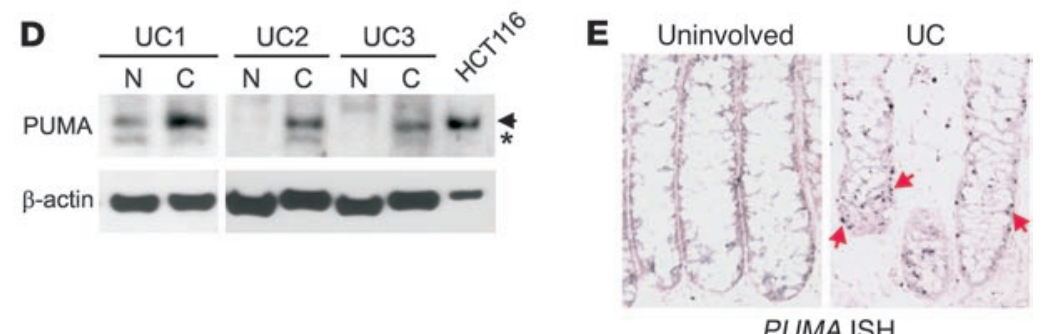

PUMAISH

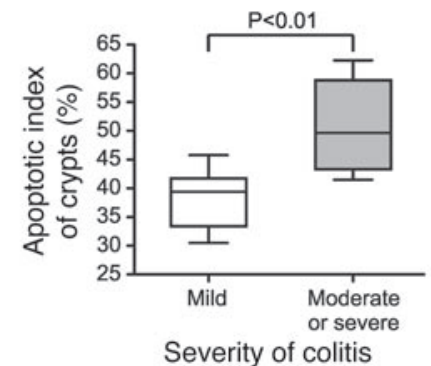

\section{Figure 1}

Apoptosis and PUMA induction in human UC specimens. (A) TUNEL (brown) staining of a matched pair of uninvolved colonic and colitis tissues from a patient with UC (original magnification, $\times 400$ ). Arrows indicate example TUNEL-positive cells. (B) Active caspase-3 (brown) staining of a matched pair of uninvolved colonic and colitis tissues from a patient with UC (original magnification, $\times 400$ ). Arrows indicate example active caspase-3-positive cells. (C) TUNEL (green) and cytokeratin (red) double staining of a colitis specimen (referred to as UC1). Arrows indicate example TUNEL and cytokeratin double-positive cells (original magnification, $\times 200$ ). (D) Western blot analysis of PUMA expression in 3 matched pairs of uninvolved colonic $(\mathrm{N})$ and colitis $(\mathrm{C})$ tissues. Lysate of PUMA-expressing HCT116 colon cancer cells was analyzed as a positive control. The arrow indicates the active form of PUMA, and the asterisk indicates an inactive form of PUMA generated by alternative splicing. (E) PUMA mRNA expression (dark dots) in uninvolved colonic and colitis tissues was analyzed by RNA ISH (original magnification, $\times 400$ ). Arrows indicate example PUMA-expressing cells. (F) Correlation between PUMA protein expression and severity of colitis in patients with UC (left panel). Correlation between apoptosis induction and severity of colitis in patients with UC (right panel). The tops of the boxes indicate the upper quartiles of the data, and the bottoms of the boxes indicate the lower quartiles of the data. The lines within the boxes indicate the median values. The top and bottom whiskers indicate the maximum values and minimum values, respectively. Values were mean $\pm \operatorname{SD}(n=7$ in each group).

\section{Results}

Apoptosis and PUMA induction in human UC tissues. To study the role of PUMA in UC and its associated IEC apoptosis, we first analyzed 23 colitis specimens and 17 uninvolved colonic tissues from patients with UC, including 12 matched pairs from the patients without pancolitis. Signs of colonic inflammation, including submucosa edema and lymphocyte infiltration, were evident in the colitis tissues (Supplemental Figure 1A; supplemental material available online with this article; doi:10.1172/ JCI42917DS1). TUNEL staining revealed apoptosis induction in every colitis specimen compared with uninvolved samples (Figure $1 \mathrm{~A}$ and Supplemental Figure 1, A-C). Apoptosis induction was confirmed by staining for active caspase-3, a biochemical marker of apoptosis (Figure 1B and Supplemental Figure 1, A, D, and E). Costaining with the epithelial marker cytokeratin and TUNEL or active caspase-3 detected apoptosis in both epithelial and nonepithelial cells (Figure 1C and Supplemental Figure 1D).
PUMA protein expression was found to be elevated in $39.1 \%$ of colitis samples ( 9 out of 23 samples) compared with that in uninvolved tissues by Western blot analysis (Figure 1D and Supplemental Figure 2A). PUMA mRNA levels were also found to be induced in colitis specimens relative to those in uninvolved tissues by RNA in situ hybridization (ISH; Figure 1E and Supplemental Figure 2B) and by real-time RT-PCR analysis (Qiu et al., unpublished observations). Immunostaining for PUMA confirmed its induction in IECs in which apoptosis could be detected simultaneously (Supplemental Figure 2, C and D). Remarkably, elevated PUMA expression significantly correlated with the severity of colitis, with 7.9-fold median PUMA induction in samples with moderate or severe colitis, compared with 3.3-fold induction in those with mild colitis (Figure 1F). Furthermore, induction of apoptosis was also found to correlate with the severity of colitis (Figure $1 \mathrm{~F}$ ) and with PUMA induction (Supplemental Figure 2E). 

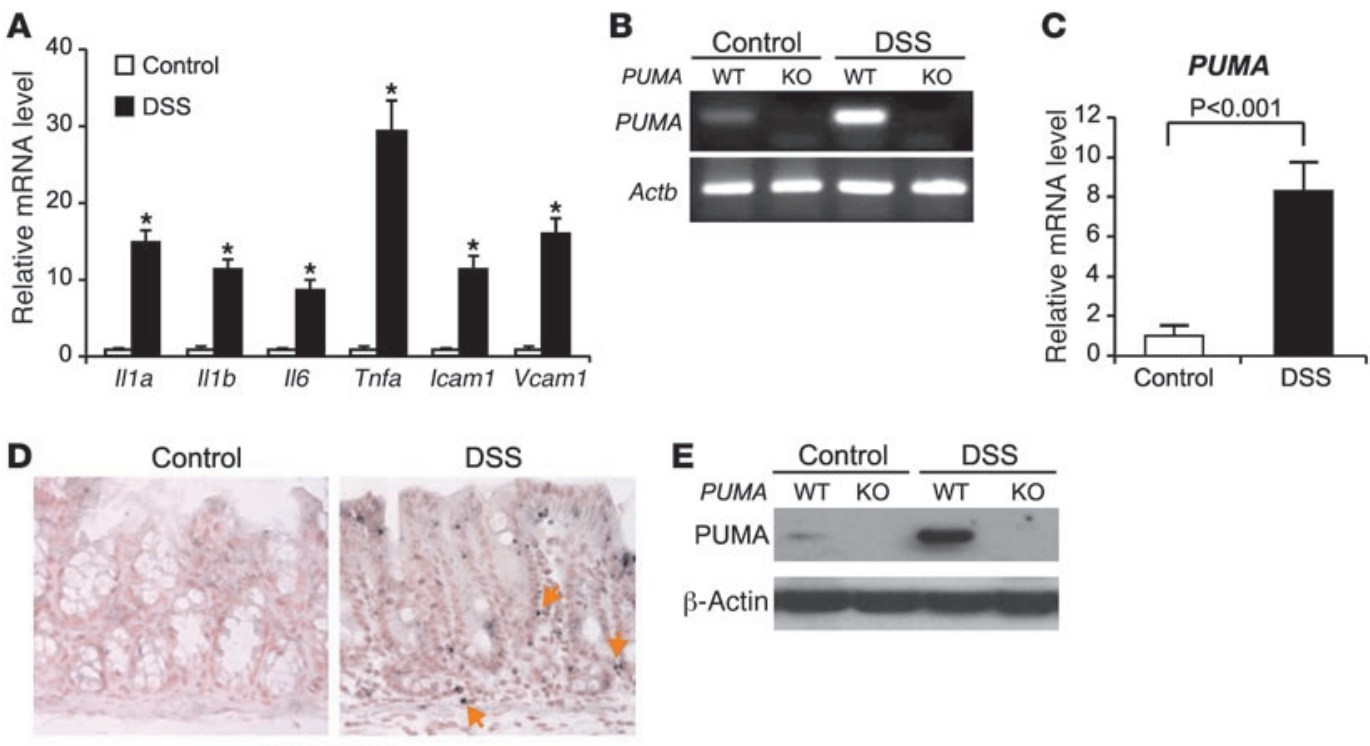

PUMA ISH

\section{Figure 2}

Induction of PUMA in colonic mucosa after DSS treatment. WT and PUMA-KO mice were treated with 5\% DSS to induce colitis. (A) The expression of the indicated inflammatory cytokines in WT mice treated with DSS for 24 hours was analyzed by real-time RT-PCR. ${ }^{*} P<0.01$ compared with the control. (B) PUMA mRNA expression in WT and PUMA-KO mice treated with DSS for 24 hours was analyzed by RT-PCR. (C) PUMA mRNA expression in WT mice treated with DSS for 24 hours was determined by real-time RT-PCR. (D) PUMA mRNA expression (dark dots) in WT mice with or without DSS treatment for 5 days was analyzed by RNA ISH (original magnification, $\times 400$ ). Arrows indicate example PUMAexpressing cells. (E) PUMA protein expression in WT and PUMA-KO mice treated with DSS for 7 days was analyzed by Western blotting. Values in $\mathbf{A}$ and $\mathbf{C}$ were mean $\pm \mathrm{SD}$ ( $n=3$ in each group).

Induction of PUMA by dextran sulfate sodium salt in mouse colonic mucosa. The above observations prompted us to use mouse models to further investigate the role of PUMA in colitis. Upon treating WT mice with 5\% dextran sulfate sodium salt (DSS), an inducer of colitis and colonic inflammation (21), we found a number of inflammatory cytokines to be induced in the colonic mucosa, including Illa, Illb, Il6, Tnfa, Icam1, and Vcam1 (Figure 2A). Similarly, PUMA mRNA levels were increased by 8 fold within 24 hours after DSS treatment (Figure 2, B and C). The induction of PUMA mRNA by DSS was confirmed by ISH analysis (Figure 2D). PUMA protein was also substantially induced after treatment (Figure 2E and Supplemental Figure 3A) and was found to be colocalized with cytokeratin (Supplemental Figure 3B). Furthermore, increased PUMA expression could be detected in purified IECs from DSS-treated mice (Supplemental Figure 4, A and B). In addition to PUMA, BH3-only proteins Noxa and $\mathrm{Bim}$, along with antiapoptotic proteins $\mathrm{Bcl}-2$ and $\mathrm{Bcl}-\mathrm{X}_{\mathrm{L}}$, were also induced by DSS (Supplemental Figure 4C). Thus, PUMA induction in IECs appears to constitute a rapid response in DSSinduced colitis and colonic inflammation.

Suppression of DSS-induced colitis in PUMA-KO mice. DSS-induced colitis is characterized by body weight loss, presence of occult or gross blood per rectum, and tissue damage (21). The disease phenotypes can be measured by the disease activity index, which is the sum of body weight, diarrhea, and rectal bleeding scores (22). To determine whether the induction of PUMA contributes to colitis, WT and PUMA-KO mice were subjected to DSS treatment. Although all DSS-treated mice developed colitis starting from day 3 (Supplemental Figure 5, A and B), the disease activity index score was significantly lower in PUMA-KO mice than in WT mice (Figure 3A). Fewer and smaller colonic ulcers were detected in PUMA-KO mice compared with WT mice after DSS treatment for 7 days (Figure 3, B-D). Histological analysis revealed substantially less epithelial damage and disruption of crypt architecture in PUMA-KO mice than in WT mice (Figure 3, E and F). However, similar levels of inflammatory cell infiltration were found in both WT and PUMA-KO mice (Figure 3E). Induction of myeloperoxidase (MPO) activity, which is an indicator of neutrophil infiltration, was also similar in WT and PUMA-KO mice after DSS treatment (Supplemental Figure 5C). These results suggest that PUMA contributes to DSS-induced colitis and intestinal damage after the initiation of the inflammatory response.

Suppression of DSS-induced IEC apoptosis by PUMA deficiency. To determine whether PUMA is involved in DSS-induced colitis through induction of apoptosis, we compared apoptosis induction in DSS-treated WT and PUMA-KO mice. DSS treatment induced DNA laddering, a classical marker of apoptosis, in the mucosa of WT mice but not in that of PUMA-KO mice (Figure 4A). DNA fragmentation quantified by diphenylamine assay was decreased from $22.9 \%$ in WT mice to $8.8 \%$ in PUMA-KO mice (Supplemental Figure 6A). Apoptosis detected by TUNEL staining started as early as 1 day after DSS treatment (Supplemental Figure 6B), primarily in the crypt areas (Qiu et al., unpublished observations). Apoptosis in these areas was sustained throughout the course of colitis and was blocked by $60 \%-80 \%$ in PUMA-KO mice (Figure 4, B and C, and Supplemental Figure 6B). Cytokeratin and TUNEL double staining confirmed the epithelial origin of the PUMA-dependent apoptosis in the crypt areas (Figure 4D and Supplemental Figure 6C). DSS treatment for 7 days also induced apoptosis in the submucosa areas, including regions containing infiltrating lymphocytes, but this apoptosis seemed to be PUMA independent (Figure 4B). 
A
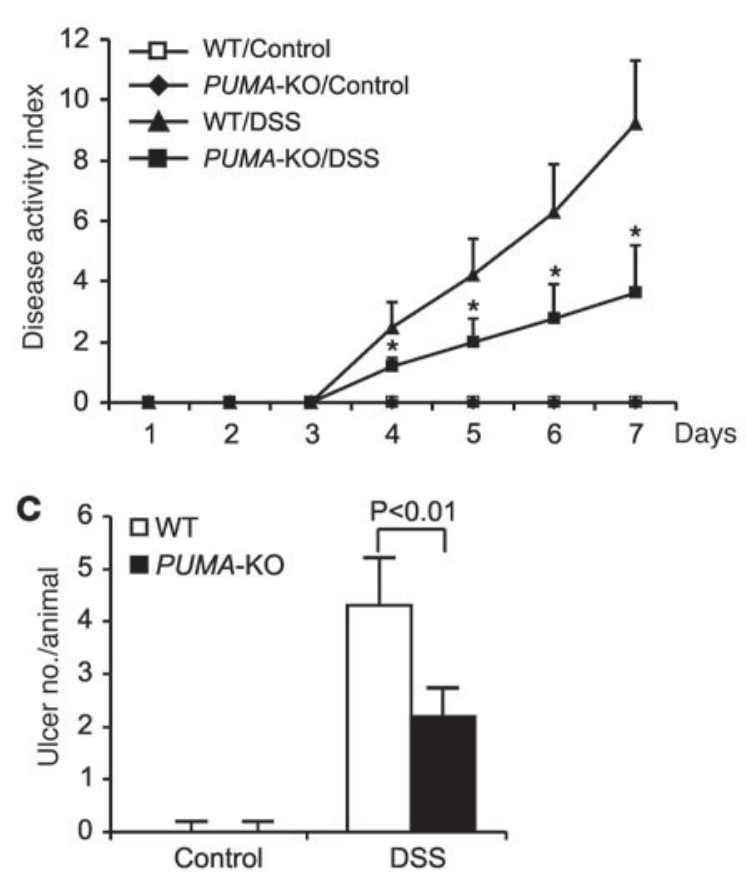

E

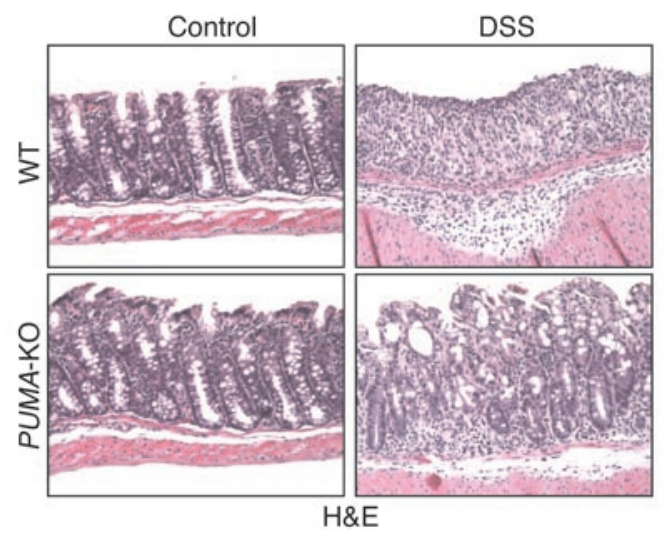

B
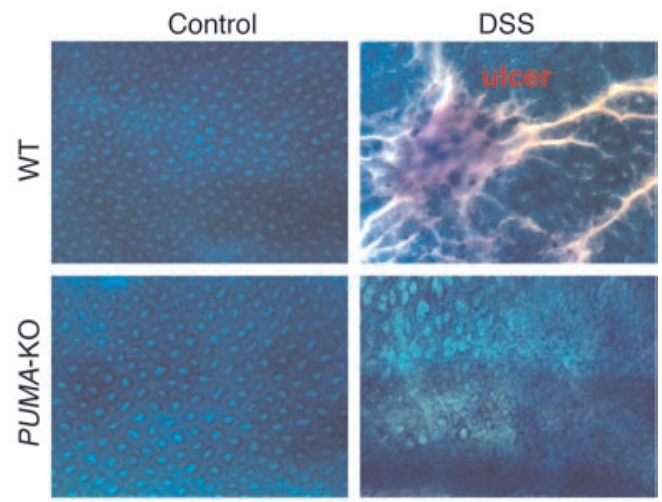

D
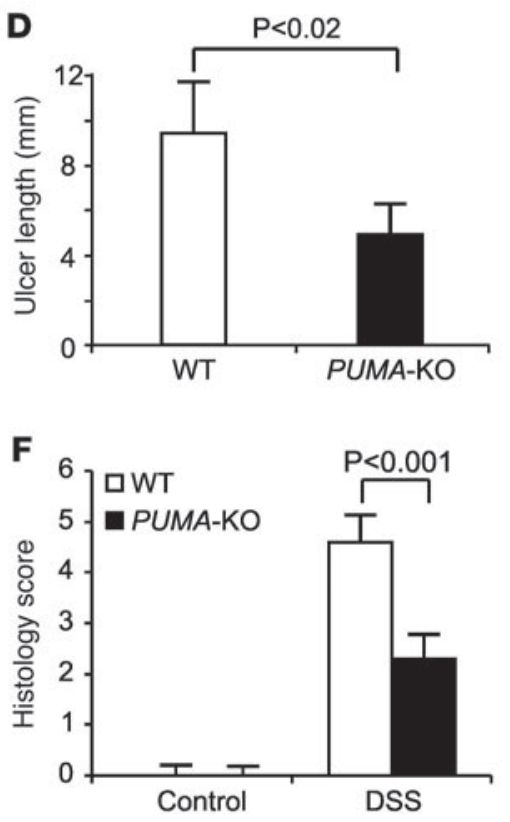

\section{Figure 3}

Suppression of DSS-induced colitis in PUMA-KO mice. WT and PUMA-KO mice were treated with 5\% DSS to induce colitis. (A) The disease activity index was determined at indicated time points, as described in the Methods. ${ }^{*} P<0.01$ compared with WT mice (2-way ANOVA). (B) Methylene blue staining of colonic tissues from WT and PUMA-KO mice after DSS treatment for 7 days (original magnification, $\times 100$ ). (C) Colonic ulcers were counted after methylene blue staining as in B. (D) Length of the colonic ulcers in WT and PUMA-KO mice after DSS treatment for 7 days. (E) H\&E staining of colonic tissues from WT and PUMA-KO mice after DSS treatment for 7 days (original magnification, $\times 200)$. (F) Histological damage after DSS treatment for 7 days was scored after H\&E staining as in $\mathbf{E}$. Values in $\mathbf{A}, \mathbf{C}, \mathbf{D}$, and $\mathbf{F}$ were mean $\pm \mathrm{SD}$ ( $n=6$ in each group).

PUMA promotes apoptosis by activating caspases through a mitochondrial pathway (13). DSS treatment of WT mice led to cytosolic accumulation of cytochrome $c$, a mitochondrial apoptogenic protein (Figure 4E). The release of cytochrome $c$ was decreased in PUMA-KO mice (Figure 4E). Concordantly, activation of caspase-3, a key caspase in the execution of apoptosis, was significantly reduced in PUMA-KO mice, as shown by the abundance of the caspase- 3 cleavage fragment (Figure 4F) and by the analysis of caspase-3 activity (Supplemental Figure 6D). Collectively, these results indicate that PUMA mediates IEC apoptosis in DSSinduced colitis through the mitochondrial pathway.
Requirement of PUMA for 2,4,6-trinitrobenzene sulfonic acidinduced colitis and IEC apoptosis. To verify the role of PUMA in colitis and IEC apoptosis, we analyzed an independent colitis model induced by 2,4,6-trinitrobenzene sulfonic acid (TNBS), which resembles CD (23). Similar to the DSS treatment, TNBS treatment also induced PUMA mRNA and protein in IECs of WT mice (Figure 5, A-C, Supplemental Figure 3, A and B, and Supplemental Figure 4, A and B). TNBS treatment induced similar levels of MPO activity and expression of Il1a, Il6, and Tnfa in both WT and PUMA-KO mice (Supplemental Figure 7, A and B). TNBS-induced colitis and colonic damage were substantially 


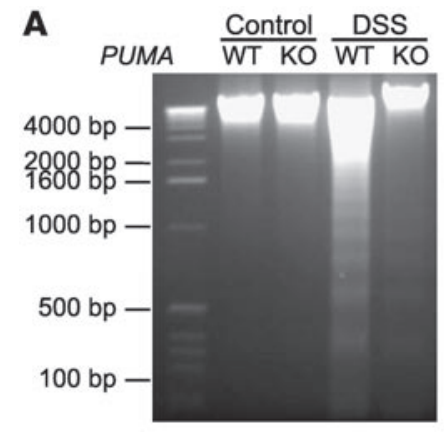

B
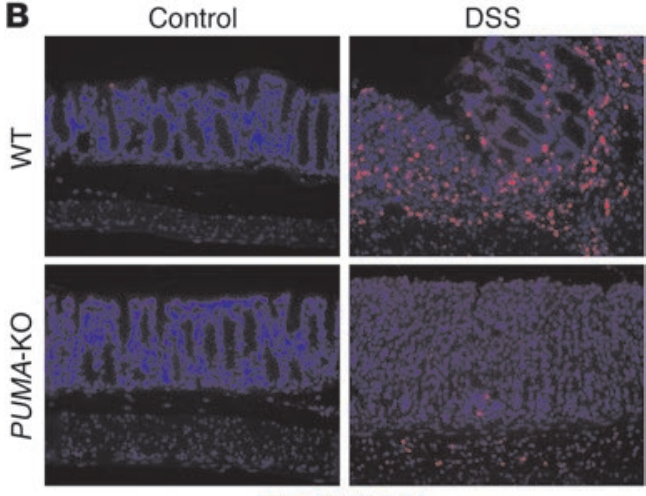

TUNEL/DAPI

C

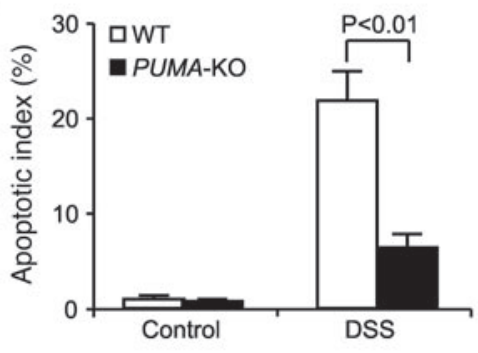

D

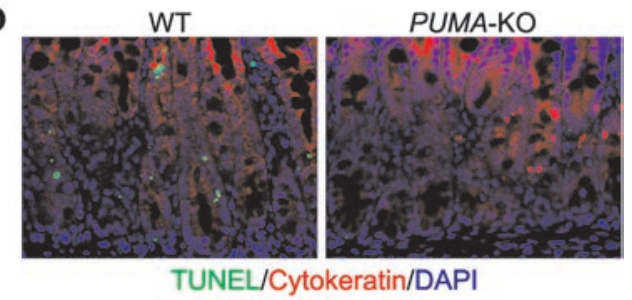

TUNEL/Cytokeratin/DAPI

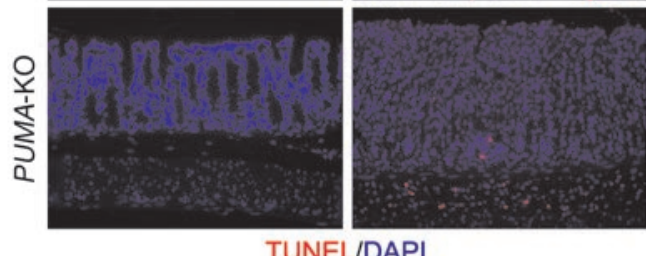

WT

Figure 4

Inhibition of DSS-induced IEC apoptosis in PUMA-KO mice. After treatment of WT and PUMA-KO mice with $5 \%$ DSS for 7 days, several methods were used to analyze apoptosis. (A) Genomic DNA isolated from colonic mucosa was analyzed by agarose gel electrophoresis. (B) TUNEL staining of colonic tissues from the treated mice (original magnification, $\times 200$ ). (C) The apoptotic index was measured by counting TUNEL signals in 100 randomly selected crypts. Values were mean $\pm \mathrm{SD}$ ( $n=3$ in each group). (D) TUNEL (green) and cytokeratin (red) double staining of colonic tissues from the treated mice (original magnification, $\times 400)$. (E) Cytochrome $c$ release was analyzed by probing the cytosolic and mitochondrial fractions of colonic mucosa using Western blotting. $\beta$-Actin and Cox IV, which are expressed in cytosol and mitochondria, respectively, were used as the controls for loading and fractionation. (F) Active caspase-3 was probed by Western blotting.

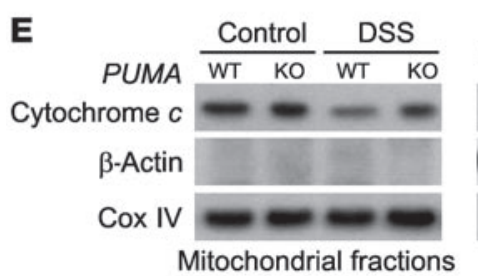

Mitochondrial fractions

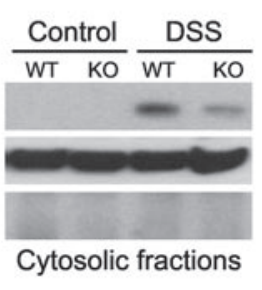

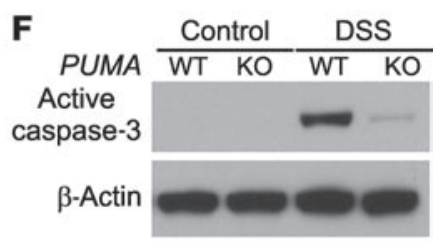

decreased in PUMA-KO mice compared with those in WT mice (Figure 5, D-F). TNBS-induced IEC apoptosis was also markedly reduced in PUMA-KO mice (Figure 5, G and H, and Supplemental Figure 6E). These results confirm the critical role of PUMA in colitis and its associated IEC apoptosis.

p53-independent PUMA induction and p53- and Bid-independent colitis. PUMA is a downstream target of p53 and plays a critical role in p53-mediated apoptosis (13). WT and p53-deficient ( $p 53-\mathrm{KO})$ mice were therefore analyzed to determine the role of p53 in DSS-induced and PUMA-mediated colitis and IEC apoptosis. After DSS treatment, the expression of p53 was unchanged in WT mice, and the induction of PUMA remained intact in p53-KO mice (Figure 6A). Deficiency in p53 did not protect mice from DSS-induced body weight loss, diarrhea, and rectal bleeding (Figure 6B). Tissue damage measured by the histological score, and the number of ulcers was also unchanged in p53-KO mice (Figure 6C and Supplemental Figure 8, A-C). Similar levels of IEC apoptosis and caspase-3 activation were detected in DSS-treated WT and p53-KO mice (Figure 6D and Supplemental Figure 8, D-F). Furthermore, TNBS-induced PUMA expression, colitis, and IEC apoptosis were also found to be completely p53 independent (Figure 6, E and F, and Supplemental Figure 9, A-C). Therefore, the effects of PUMA on colitis and IEC apoptosis were not attributable to p53. To further determine the potential role of other $\mathrm{BH} 3$-only proteins in colitis, mice deficient in the BH3-only protein Bid (Bid-KO mice) were analyzed. We found that colonic damage and IEC apoptosis induced by DSS or TNBS were not different in WT and Bid-KO mice (Supplemental Figure 10, A-H).

Effects of TNF inbibition on PUMA induction and colitis. Our recent study showed that PUMA can be induced by TNF- $\alpha$ through an NF-кBdependent but p53-independent mechanism (18). This prompted us to test whether NF- $\mathrm{BB}$ and TNF are involved in PUMA induction in DSS- and TNBS-induced colitis. Indeed, administration of the NF- $\kappa B$ inhibitor, Bay 117082, blunted the induction of PUMA in DSS-treated WT mice, in conjunction with reduced activation (phosphorylation)

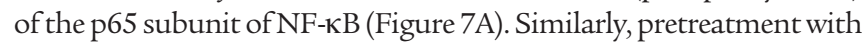
infliximab (Remicade), an anti-TNF antibody clinically used for IBD treatment, also suppressed DSS-induced PUMA expression and p65 phosphorylation (Figure 7B and Supplemental Figure 11A), suggesting that the induction of PUMA by DSS is mediated by TNF through NF- $\kappa$ B. In addition to PUMA induction, TNF inhibition, an important means for treatment of IBD (1), suppressed DSS-induced colitis (Figure 7C), colonic damage (Figure 7D and Supplemental Figure 11B), ulcer formation (Supplemental Figure 11C), and IEC apoptosis (Figure 7, E and F). The extent of suppression conferred by TNF inhibition was similar to that observed in PUMA-KO mice.

Consistent with the findings from the DSS model, the induction of PUMA by TNBS was also reduced following NF- $\kappa B$ inhibition (Figure 8A). Cotreatment with infliximab compromised TNBS-induced PUMA 
A

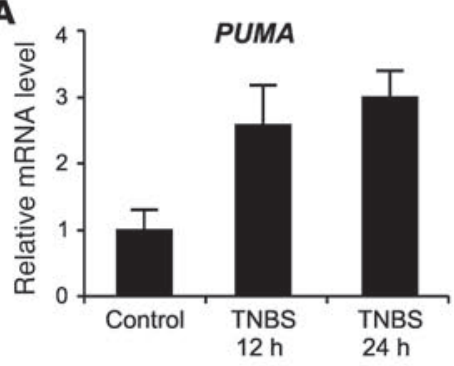

D

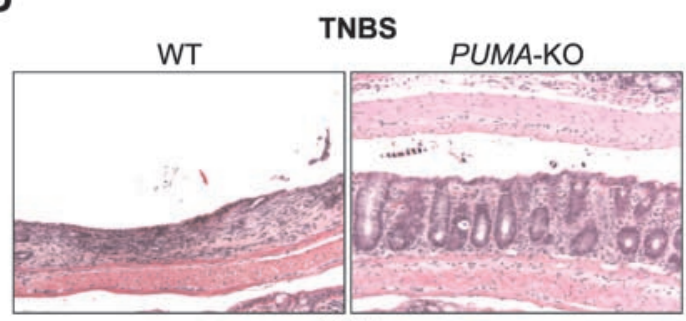

H\&E

G

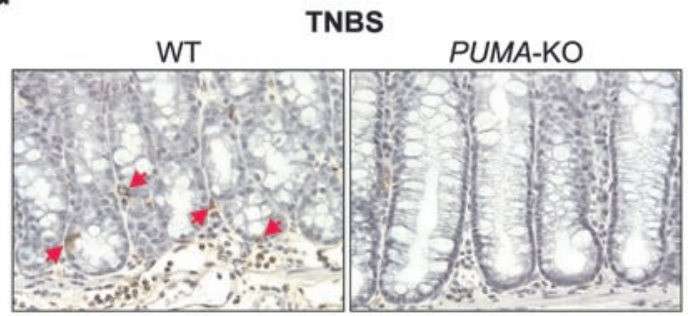

TUNEL
B

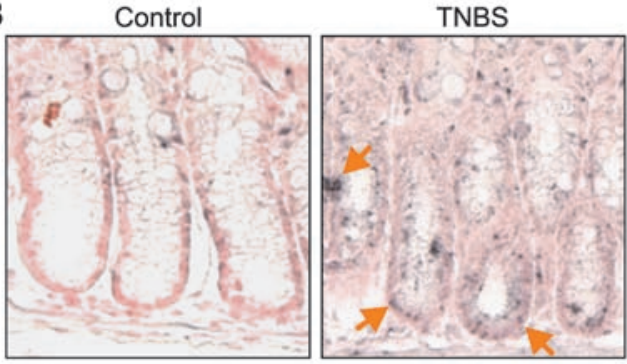

PUMA ISH
C

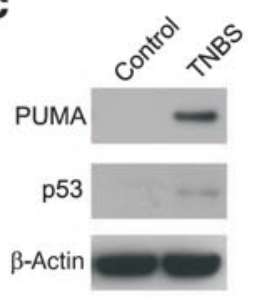

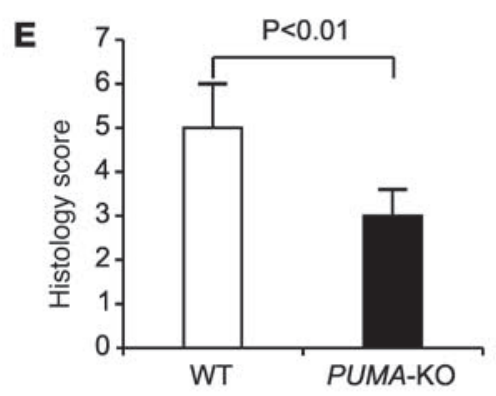

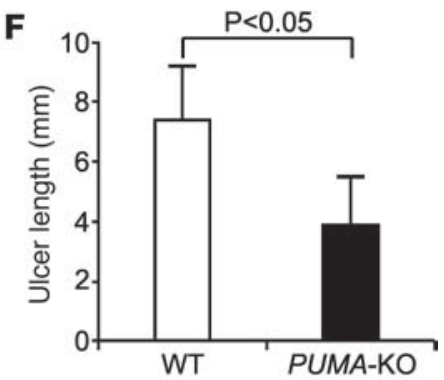

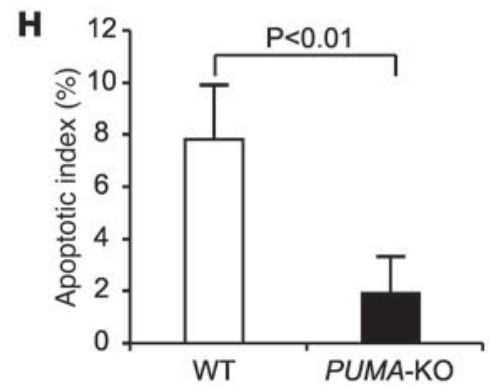

Figure 5

TNBS-induced and PUMA-dependent colitis. WT and PUMA-KO mice were treated with $100 \mathrm{mg} / \mathrm{kg}$ of TNBS to induce colitis. (A) PUMA mRNA expression in colonic mucosa of WT mice treated with TNBS for 12 or 24 hours was analyzed by real-time RT-PCR. (B) PUMA mRNA expression (dark dots) in WT mice with or without TNBS treatment for 3 days was analyzed by RNA ISH (original magnification, $\times 400$ ). Arrows indicate example PUMA-expressing cells. (C) PUMA and p53 protein expression in WT mice after TNBS treatment for 3 days was determined by Western blotting. (D) H\&E staining of colonic tissues from the mice treated with TNBS for 3 days (original magnification, $\times 200$ ). (E) Histological damage in mice treated with TNBS for 3 days was scored after H\&E staining as in $\mathbf{D}$. (F) Length of the colonic ulcers in WT and PUMA-KO mice treated with TNBS for 3 days. (G) TUNEL (brown) staining of colonic tissues from mice treated with TNBS for 3 days (original magnification, $\times 200$ ). Arrows indicate example TUNEL-positive cells. $(\mathbf{H})$ The apoptotic index was calculated by counting TUNEL spots in 100 randomly selected crypts. Values in $\mathbf{A}, \mathbf{E}, \mathbf{F}$, and $\mathbf{H}$ were mean $\pm \operatorname{SD}(n=3$ in each group).

expression and 655 phosphorylation (Figure 8B), colonic damage (Figure 8C and Supplemental Figure 12A), and IEC apoptosis (Figure $8 \mathrm{D}$ and Supplemental Figure 12, B and C). Furthermore, pretreatment with the TNF inhibitor pentoxifylline also inhibited colitis and IEC apoptosis induced by DSS (Supplemental Figure 13, A-I) and those induced by TNBS (Supplemental Figure 14, A-E). However, NF-кB inhibition by pretreating mice with Bay 117082 did not protect against DSS- or TNBS-induced colitis and IEC apoptosis (Figure 9). Collectively, these results suggest that inhibition of PUMA expression contributes to the antiinflammatory effects of TNF inhibitors.

\section{Discussion}

Apoptotic cell death has been implicated as a major homeostatic and pathogenic mechanism of intestinal epithelium (2). In this study, we found that increased PUMA expression is correlated with the severity of colitis and induction of apoptosis in human UC samples. PUMA deficiency in mice abrogated DSS- and TNBS-induced colitis and IEC apoptosis, supporting the hypothesis that PUMA functions as a critical mediator of IEC apoptosis and a significant modulator of UC. The functional role of PUMA in IBDs needs to be validated using other cohorts of patient samples. The broader implications of our study can be verified using other colitis models, such as the IL10-deficient genetic model. Whether PUMA-mediated IEC apoptosis is responsible for disrupting the barrier function of the intestinal epithelial monolayer remains to be determined. PUMA has recently been shown to regulate $\mathrm{T}$ cell apoptosis (24). It is possible that PUMA may contribute to colitis by affecting $\mathrm{T}$ cell apoptosis, which is known to be abnormally regulated in IBDs $(25,26)$. In addition to PUMA, other BH3-only proteins, such as Noxa and Bim, may also be involved in colitis (27).

The induction of PUMA mRNA and protein occurred within 12-24 hours after DSS or TNBS treatment and could be detected throughout the treatment. This rapid and sustained PUMA induction 
A

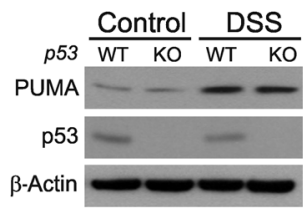

B

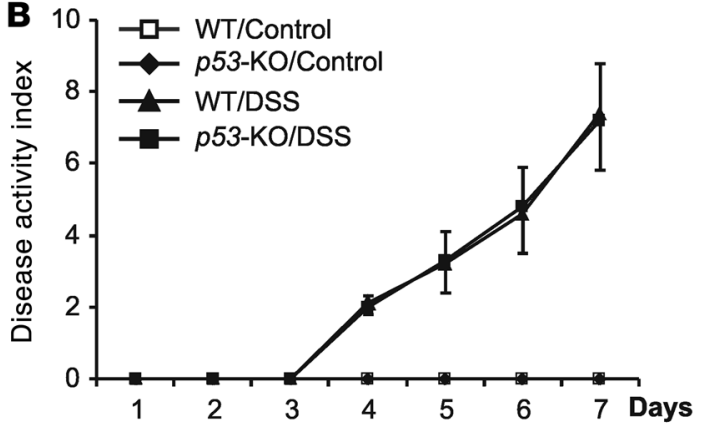

C

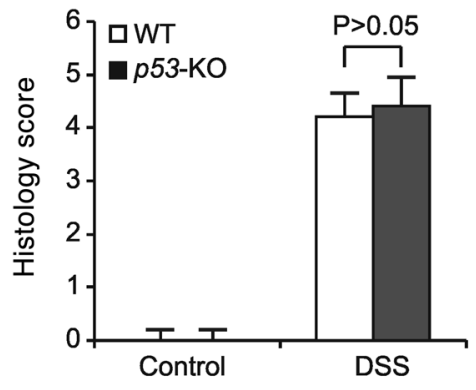

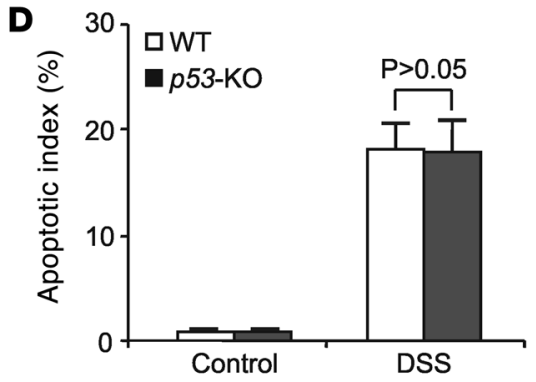

$\mathbf{E}$

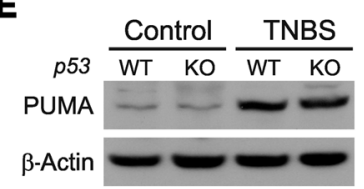

$\mathbf{F}$

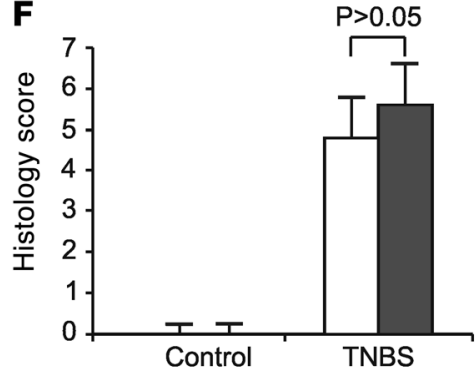

Figure 6

p53-independent PUMA induction and colitis after DSS or TNBS treatment. WT and $p 53-\mathrm{KO}$ mice were treated with $5 \%$ DSS or $100 \mathrm{mg} / \mathrm{kg}$ of TNBS to induce colitis. (A) PUMA and p53 expression in colonic mucosa from the mice treated with DSS for 24 hours was analyzed by Western blotting. (B) The disease activity index was measured at the indicated time points after DSS treatment. (C) Histological damage was scored after H\&E staining of colonic tissues from the mice treated with DSS for 7 days. (D) The apoptotic index was calculated by counting TUNEL signals in 100 crypts sections from the mice treated with DSS for 7 days. (E) PUMA expression in colonic mucosa from the mice treated with TNBS for 24 hours was analyzed by Western blotting. (F) Histological damage was scored after H\&E staining of colonic tissues from the mice treated with TNBS for 7 days. Values in $\mathbf{B}-\mathbf{D}$ and $\mathbf{F}$ were mean $\pm \mathrm{SD}(n=5$ in each group).

seems to be a downstream inflammatory response, as cytokine induction and lymphocyte infiltration could still be detected in PUMA-KO mice. The induction of PUMA by DSS and TNBS is driven, at least in part, by TNF- $\alpha$, which is the major cytokine that initiates and perpetuates intestinal mucosal inflammation and damage (28). Interestingly, Il6 and Tnfa expression was reduced in PUMA$\mathrm{KO}$ mice compared with that in WT mice after TNBS treatment (Supplemental Figure 7A), suggesting a feedback loop in which PUMA-mediated apoptosis further enhances cytokine production. Our previous studies have already shown that deficiency in the p 65 component of NF- $\kappa B$, a key regulator of intestinal inflammation (17), blunted PUMA induction by TNF- $\alpha$ in vitro and in vivo (18). This observation, along with the finding that NF- $\mathrm{KB}$ or TNF inhibition suppressed PUMA induction by DSS or TNBS, suggests that the TNF/NF- $\mathrm{KB}$ axis is responsible for PUMA induction and subsequent IEC apoptosis in colitis. Potential involvement of other cytokines is also possible, as suggested by a recent study, showing PUMA induction by interferon- $\gamma$ through the transcription factor IRF-1 (29). Unlike PUMA or TNF inhibition, NF- $\mathrm{B}$ blockage did not alleviate colitis, probably reflecting the ability of NF- $\mathrm{KB}$ to activate both proapoptotic proteins, such as PUMA, and antiapoptotic proteins, such as Bcl-2 and Bcl- $\mathrm{X}_{\mathrm{L}}$ (Supplemental Figure 4C).

p53 is absolutely necessary for PUMA induction in IECs by DNA-damaging agents such as $\gamma$-irradiation (19). It also activates several proapoptotic proteins implicated in IBDs, such as Fas, death receptor 4 (DR4), and DR5 (30). Surprisingly, DSSand TNBS-induced PUMA expression and colitis are completely p53-independent. p53 is also unnecessary for the induction of PUMA in response to intestinal ischemia/reperfusion (20). However, our study was focused on acute changes at early time points, whereas chronic colitis has been shown to induce DNA damage and trigger a p53 response (31). FoxO3A, a transcription factor that induces PUMA in response to cytokine deprivation (32), is not required for PUMA induction by TNF- $\alpha$ (18). Our results seem to reveal what we believe to be a novel apoptotic pathway through the TNF- $\alpha /$ NF- $\mathrm{KB} /$ PUMA axis, which may play a broad functional role in tissue inflammatory response and damage. It will be interesting to test whether this pathway is also involved in mucosal inflammation induced by bacterial or viral infection.

Modulation of PUMA expression may contribute to the effectiveness of current IBD treatment regimens, such as anti-TNF (33). TNF inhibitory agents may block IEC apoptosis by inhibiting PUMA expression. Our recent studies show that suppressing PUMA expression using an antisense approach significantly improved animal survival and protected against intestinal damage induced by high doses of $\gamma$-irradiation (19). Growth factors, such as insulin-like growth factor 1 and basic fibroblast growth factor, also alleviate radiation-induced intestinal injury, at least in part, by suppressing PUMA (34). Therefore, PUMA inhibition may offer an effective way to protect against IEC apoptosis and thereby may serve as a novel anti-IBD strategy. In conclusion, our study has demonstrated that PUMA contributes to colitis by promoting IEC apoptosis and may be a useful target for developing new IBD treatment options. 


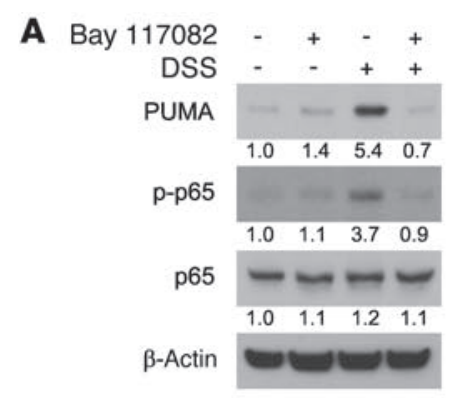

D

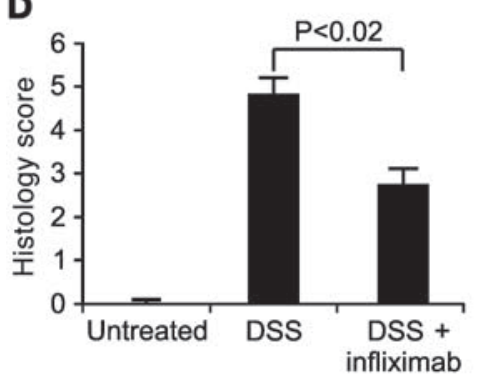

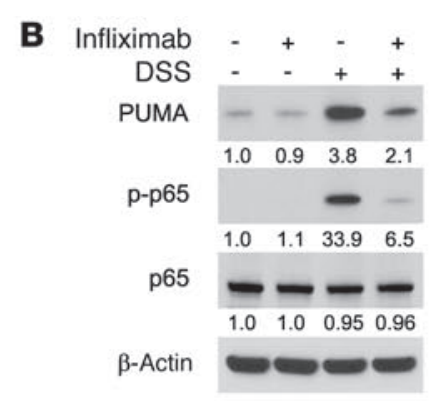

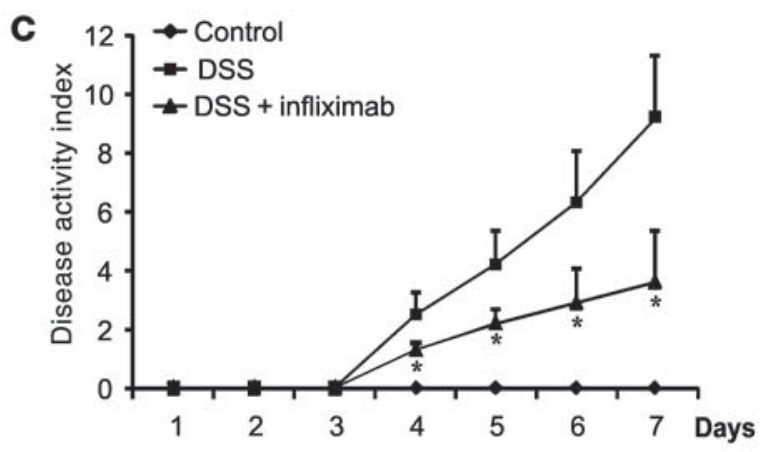

$\mathbf{E}$

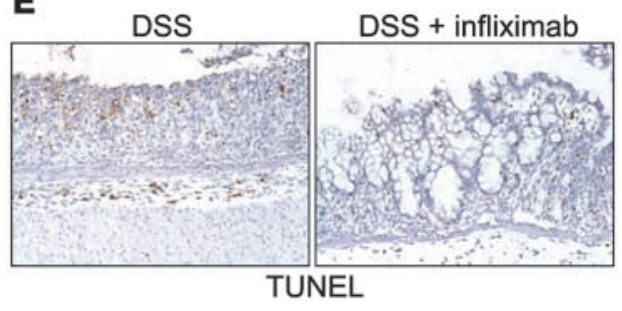

$\mathbf{F}$

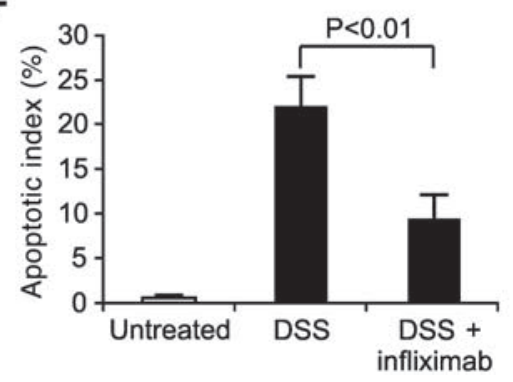

Figure 7

Effects of TNF inhibition on DSS-induced PUMA expression and colitis. (A) WT mice were treated with $5 \%$ DSS, alone or in combination with $8 \mathrm{mg} / \mathrm{kg}$ of the NF-кB inhibitor Bay 117082. The expression of the indicated proteins in colonic mucosa was analyzed by Western blotting. p-p65, phosphorylated p65. (B) WT mice were treated with 5\% DSS, alone or in combination with $10 \mathrm{mg} / \mathrm{kg}$ of the TNF antibody infliximab, for 24 hours. PUMA protein expression in the colonic mucosa of the treated mice was analyzed by Western blotting. (C) The disease activity index of the mice treated with DSS, with or without infliximab, was measured at indicated time points. ${ }^{*} P<0.01$ compared with DSS alone (2-way ANOVA). (D) Histological damage in the colonic tissues from the mice treated with DSS, with or without infliximab, for 7 days was scored after H\&E staining. (E) TUNEL (brown) staining of colonic tissues from the mice treated as indicated for 7 days (original magnification, $\times 200$ ). (F) The apoptotic index was calculated by counting TUNEL signals in 100 crypts. In A and B, relative expression of each sample, normalized to that of the loading control $\beta$-actin, is indicated, with that of the untreated animal arbitrarily set as 1.0. Values in C, D, and F were mean \pm SD ( $n=5$ in each group).

\section{Methods}

Tissue samples. Frozen specimens of 23 colitis and 17 uninvolved tissues, including 12 matched pairs of samples from patients with UC without pancolitis, were obtained from the Digestive Disease Tissue Resource of the University of Pittsburgh. The acquisition of tissue samples was approved by the Institutional Review Board at the University of Pittsburgh. Written informed consent was received from each patient prior to inclusion in the study. Twelve males and eleven females were represented in colitis group, with the group age ranging from 10-70 years old, whereas 8 males and 9 females were represented in uninvolved group, with the group age ranging from $20-70$ years old. Among the 23 patients in the UC group, colitis was found in the colons of 20 patients, the rectums of 10 patients, the ceca of 2 patients, the duodenum of 1 patient, and the terminal ileum of 1 patient. Among the 12 patients whose matched pairs of samples were analyzed, 5 were treated with steroids, 2 received immunomodulator treatment, and 1 was subjected to anti-TNF therapy.

Mice and treatment. All animal experiments were approved by the Institutional Animal Care and Use Committee at the University of Pittsburgh. Mice in the C57BL/ 6 background with different genotypes, including WT, PUMA-KO (PUMA-/-), Bid-KO ( $\left.\mathrm{Bid}^{-/-}\right)$, and $p 53-\mathrm{KO}\left(p 53^{-/-}\right)$, were generated by breeding and identified by PCR genotyping, as previously described (19, $20,35)$. The mice were housed in microisolator cages and allowed access to water and chow ad libitum.

Six- to ten-week-old littermates were treated with either DSS or TNBS to induce colitis. For DSS treatment, mice were fed with 5\% DSS (MP Biomedicals) in drinking water. Mice received DSS treatment for 1, 3, 5, or 7 days and were immediately sacrificed after treatment. For TNBS treatment, mice were presensitized on the skin for 1 week with TNBS (Sigma-Aldrich) at $40 \mathrm{mg} / \mathrm{kg}$ body weight in $50 \%$ ethanol. After fasting overnight, the presensitized mice received $100 \mathrm{mg} / \mathrm{kg}$ TNBS by rectal injection with a $1-\mathrm{ml}$ syringe fitted with a catheter, as previously described (36). Sacrifice of mice was performed 3 days after TNBS treatment. For TNF inhibition, mice were i.p. injected at 1 hour prior to DSS or TNBS treatment with the anti-TNF antibody infliximab (Remicade; Centocor Ortho Biotech) at $10 \mathrm{mg} / \mathrm{kg}$ or the TNF inhibitor pentoxifylline (Sigma-Aldrich) at $200 \mathrm{mg} / \mathrm{kg}$. For NF-KB inhibition, mice were injected at 1 hour prior to DSS or TNBS treatment with $8 \mathrm{mg} / \mathrm{kg}$ of the NF- $\mathrm{KB}$ inhibitor BAY 117082 (EMD Biosciences) once daily. After TNF or NF- $\mathrm{KB}$ inhibition, mice were treated with DSS or TNBS and then sacrificed using the same schedule as those treated with DSS or TNBS alone.

Analysis of $m R N A$ and protein expression. Preparation of intestinal mucosa scraping and isolation of DNA, total RNA, and protein extracts from mouse tissues were performed as previously described $(20,37)$. Real-time RT-PCR was performed on a Mini Opticon Real-time PCR System (Bio-Rad) with SYBR Green (Invitrogen). Primers for RT-PCR are listed in Supplemental Table 1, except for the previously described PUMA and $\beta$-actin primers (20). Agarose (2\%) gel electrophoresis was used to verify PCR products.

After protein extracts were subjected to NuPage gel (Invitrogen) electrophoresis, Western blotting was performed using antibodies for human PUMA (15); murine PUMA (Abcam); p53, Bax, and p65 (all from Santa Cruz Biotechnology Inc.); active caspase-3, Bcl-2, Mcl-1, and cytochrome $c$ (all from BD Biosciences); phospho-p65 (Ser536) and Bad (both from

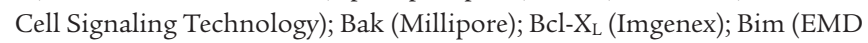
Biosciences); Noxa (Merck); Bcl-w (Enzo Life Sciences); Bid (a gift from 
A

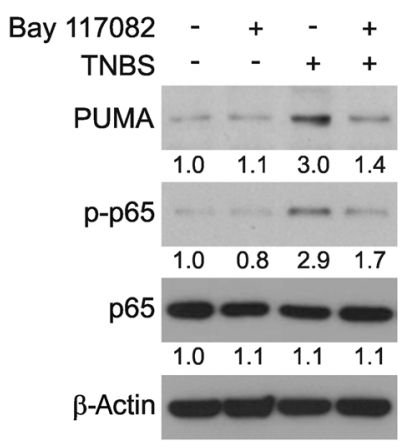

C

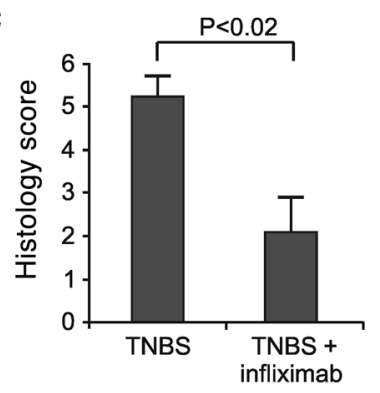

B

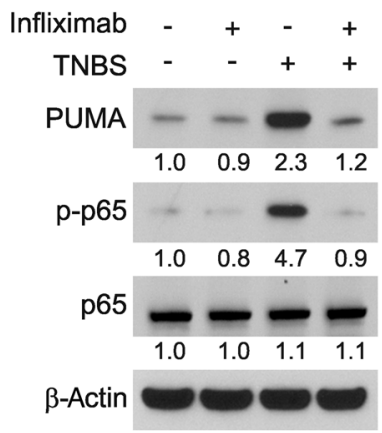

D

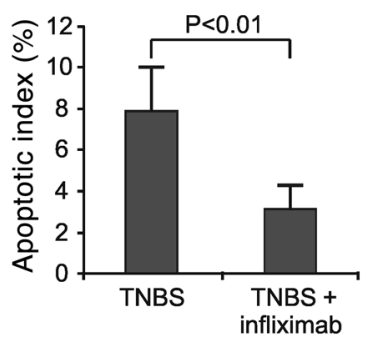

\section{Figure 8}

Effects of TNF inhibition on TNBS-induced PUMA expression and colitis. (A) WT mice were treated for 3 days with $100 \mathrm{mg} / \mathrm{kg}$ TNBS, alone or in combination with $8 \mathrm{mg} / \mathrm{kg}$ of the NF-kB inhibitor Bay 117082 . The expression of the indicated proteins in colonic mucosa was analyzed by Western blotting. (B) WT mice were treated for 3 days with $100 \mathrm{mg} / \mathrm{kg}$ TNBS, alone or in combination with $10 \mathrm{mg} / \mathrm{kg}$ of the TNF antibody infliximab. The expression of the indicated proteins in colonic mucosa was analyzed by Western blotting. (C) Histological damage in the mice treated with TNBS, with or without infliximab, for 3 days was quantified after H\&E staining. Values were mean $\pm \mathrm{SD}(n=3$ in each group). (D) The apoptotic index in the mice treated as indicated for 3 days was calculated by counting TUNEL signals in 100 randomly selected crypts. Values were mean $\pm \operatorname{SD}(n=3$ in each group). In $\mathbf{A}$ and $\mathbf{B}$, relative expression of each sample, normalized to that of the loading control $\beta$-actin, is indicated, with that of the untreated animal arbitrarily set as 1.0 .
Xiao-Ming Yin at Indiana University, Indianapolis, Indiana, USA); and $\beta$-actin (Sigma-Aldrich) as previously described (15). For mouse analysis, representative results of 2 to 3 mice analyzed are presented, with each lane on a Western blot representing a single animal. Western blot band intensities were quantified using ImageJ software (http://rsbweb.nih.gov/ij/).

Immunostaining. Tissue sections ( $5-\mu \mathrm{m}$ thick) were deparaffinized, rehydrated, and treated with $3 \%$ hydrogen peroxide, followed by antigen retrieval in boiling $0.1 \mathrm{M}$ citrate ( $\mathrm{pH}$ 6.0) buffer for 10 minutes once. The sections were then blocked by $20 \%$ rabbit serum for 30 minutes. Immunostaining was performed as previously described for active caspase-3 (34). PUMA staining was performed at $4^{\circ} \mathrm{C}$ overnight using a mouse anti-PUMA antibody (SigmaAldrich), with Alexa Fluor 594 (Invitrogen) for signal detection. Cytokeratin staining was done at $4{ }^{\circ} \mathrm{C}$ overnight using a mouse anti-cytokeratin antibody (Abcam), with Alexa Fluor 488 (Invitrogen) or Alexa Fluor 594 for signal detection. For double staining, TUNEL staining was performed after PUMA or cytokeratin staining. Cytokeratin staining was performed prior to PUMA or active caspase- 3 staining. Cells with positive staining were scored in at least 100 crypt sections and reported as mean \pm SD.

RNA ISH. Probe template was prepared by RT-PCR amplifying a 398-bp PUMA cDNA fragment with one of the primers containing a T7 promoter sequence at $5^{\prime}$. Digoxigenin-labeled (DIG-labeled) RNA probes were transcribed using the DIG RNA Labeling Kit (SP6/T7) (Roche Diagnostics). Paraffin-embedded tissue was sectioned ( 5 - $\mu \mathrm{m}$ thick), rehydrated, denatured with $0.2 \mathrm{~N} \mathrm{HCl}$, treated with Proteinase $\mathrm{K}$ (Ambion), postfixed in $4 \%$ paraformaldehyde, and acetylated. After a 1-hour prehybridization at $60^{\circ} \mathrm{C}$, denatured DIG-labeled probes were hybridized to sections at $60^{\circ} \mathrm{C}$ for 16 to 24 hours. Hybridization buffer contained $65 \%$ formamide, $5 \mathrm{X}$ SSC, $2 \%$ Roche blocking powder, $1 \mu \mathrm{g} / \mathrm{ml}$ yeast tRNA, $50 \mu \mathrm{g} / \mathrm{ml}$ heparin, 5 mM EDTA, and 0.05\% 3-([3-cholamidopropyl] dimethylammonio)-1propanesulfonate. After a number of washes to decrease salt concentration, slides were incubated with $\alpha$-DIG-horse radish peroxidase (Abcam), biotinyl tyramide (DAKO), $\alpha$-biotin-horse radish peroxidase (Abcam), and then additional biotinyl tyramide (DAKO) to amplify the signal. After incubation with $\alpha$-biotin-alkaline phosphatase (Abcam), the signal was visualized using nitroblue-tetrazolium-chloride/5-bromo-4-chlorindolyl-phosphate (Roche Diagnostics) solution, and counterstaining was applied with nuclear Fast Red (Vector Laboratories). When staining was complete, slides were washed in water and mounted in Clear Mount (Electron Microscopy Sciences).

IEC isolation. Dissected mouse colons were cut open longitudinally and incubated in 50-ml tubes with $20 \mathrm{ml} \mathrm{Ca}^{2+}$ - and $\mathrm{Mg}^{+}$-free Hank's Buffered Salt Solution (CMF-HBSS; Invitrogen) containing $10 \mathrm{mM}$ dithiothreitol and $1.5 \mathrm{mM}$ EDTA at $4{ }^{\circ} \mathrm{C}$ for 1 hour. After incubation, the tube was vigorously shaken for 2 minutes to dislodge IECs. The cell suspensions were passed through a $100-\mu \mathrm{m}$ cell strainer (BD Biosciences) and were centrifuged at room temperature at $400 \mathrm{~g}$ for 5 minutes. The cell pellet was resuspended in $2 \mathrm{ml} \mathrm{CMF-HNBSS}$ and incubated at $4^{\circ} \mathrm{C}$ for 1 hour with agitation with a pre-made mixture of $100 \mu \mathrm{l}$ TBS, $5 \mu \mathrm{l}$ rat anti-mouse epithelial cell adhesion molecule (EPCAM), and $50 \mu \mathrm{l}$ anti-rat IgG Danabeads (Invitrogen). The beads were isolated by a Magnetic Particle Concentrator (Invitrogen) and washed 3 times with CMF-HBSS. The isolated IECs were stained for EpCAM and also lysed for Western blotting.

Determination of the disease activity index score. The murine colitis phenotypes were measured by the disease activity index score, which is the sum of body weight, diarrhea, and bleeding scores determined daily during the treatment, as previously described (22). All scores were relative to the scores on day 1 , which were set as 0 . For body weight, no weight loss was scored as 0 , loss of $1 \%-5 \%$ weight registered as 1 , loss of $6 \%-10 \%$ weight registered as 2 , loss of $11 \%-20 \%$ weight registered as 3 , and loss of higher than $20 \%$ weight registered as 4 . For stool consistency, 0 was scored for well-formed stool pellets, 2 was scored for pasty and semiformed stools that did not adhere to the anus, and 4 was scored for liquid stools that adhered to the anus. For bleeding, which was analyzed by the Hemoccult fecal occult blood test (Beckman Coulter), 0 was assigned for no blood, 2 was assigned for positive Hemoccult, and 4 was assigned for gross bleeding. All scores were blindly confirmed by 2 trained individuals.

Analysis of tissue damage and histology. Entire colons were collected from the treated mice and were fixed flat between wet filter papers in $10 \%$ neutral buffered formalin for 48 hours. The tissues were stained with 
A
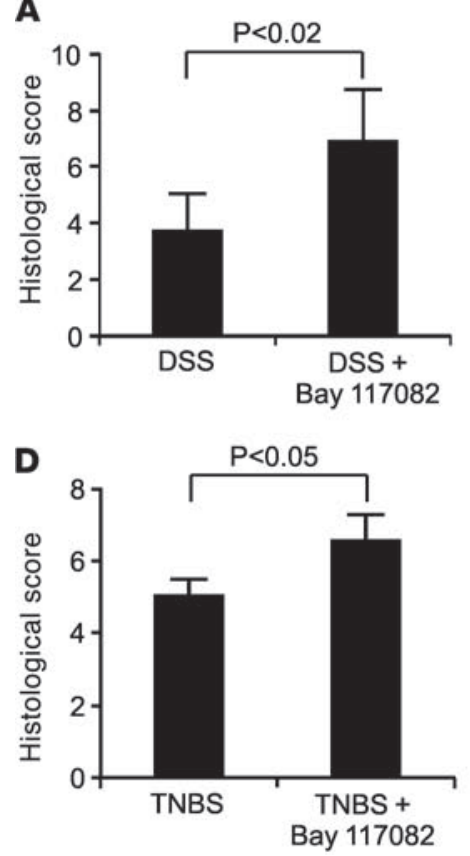

B

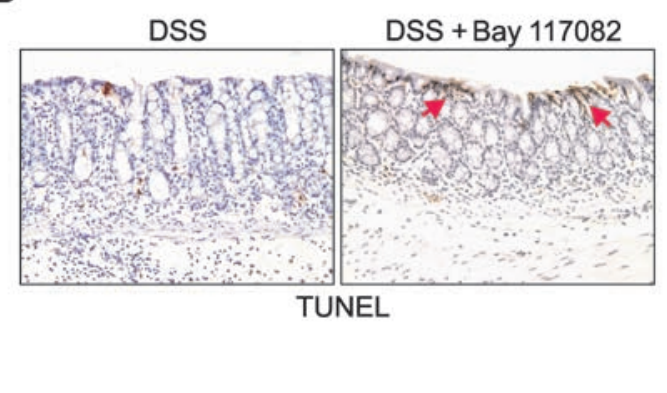

E

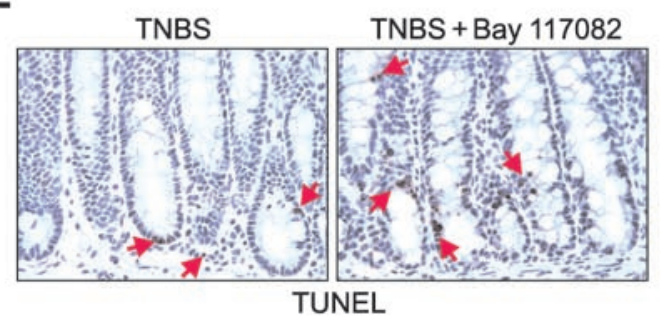

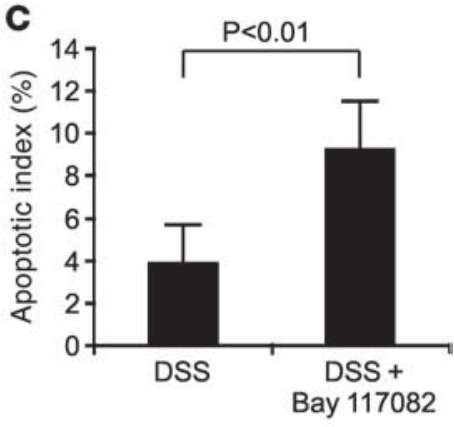

$\mathbf{F}$

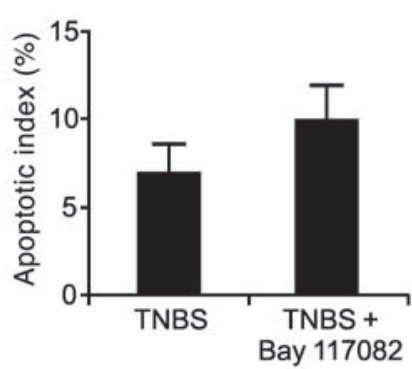

\section{Figure 9}

Effects of NF-KB inhibition on DSS- or TNBS-induced colitis. (A) WT mice were treated with $5 \%$ DSS, alone or in combination with $8 \mathrm{mg} / \mathrm{kg}$ of the NF-KB inhibitor Bay 117082, for 7 days. Histological damage was scored after H\&E staining of colonic tissues from the treated mice. (B) TUNEL (brown) staining of colonic tissues from the mice treated as in A (original magnification, $\times 200$ ). Arrows indicate example TUNEL-positive cells. (C) The apoptotic index was calculated by counting TUNEL signals in 100 randomly selected crypts after TUNEL staining as in B. (D) WT mice were treated with $100 \mathrm{mg} / \mathrm{kg}$ TNBS, alone or in combination with $8 \mathrm{mg} / \mathrm{kg}$ of the NF-kB inhibitor Bay 117082 , for 3 days. Histological damage was scored after H\&E staining of colonic tissues from the treated mice. (E) TUNEL (brown) staining of colonic tissues from the mice treated as in D (original magnification, $\times 400)$. Arrows indicate example TUNEL-positive cells. (F) The apoptotic index was calculated by counting TUNEL signals in 100 randomly selected crypts after TUNEL staining as in E. Values in A, C, D, and $\mathbf{F}$ were mean \pm SD ( $n=3$ in each group).

$0.2 \%$ methylene blue (Sigma-Aldrich) in formalin solution for 5 minutes, and ulcerous areas were counted within 24 hours under a microscope. The tissues were subsequently processed as previously described (19). Paraffin-embedded sections (5-mm thick) were subjected to H\&E staining for histological analysis. Histological scores were determined blindly based on the previously described criteria (38): 0 , normal; 1 , moderate mucosal inflammation without erosion and ulcer; 2 , severe mucosal inflammation with erosion; 3 , severe mucosal inflammation with ulcer ( $1 \mathrm{~mm})$; 4, severe mucosal inflammation with ulcer $(1-3 \mathrm{~mm}) ; 5$, severe mucosal inflammation with ulcer ( $>3 \mathrm{~mm})$.

Analysis of apoptosis. DNA laddering and fragmentation were analyzed as described previously (20). TUNEL staining was performed using the ApopTag Kit (Chemicon International) according to the manufacturer's instructions. TUNEL-positive crypts were counted in 100 randomly selected crypts. Cytochrome $c$ release was analyzed by Western blot probing of mitochondrial and cytosolic fractions isolated from intestinal mucosal scraping by differential centrifugation (20). Caspase-3 activity was measured using a colorimetric assay kit (EMD Biosciences), according to the manufacturer's instructions, or by Western blotting for active caspase-3.

Statistics. Statistical analysis was carried out using GraphPad Prism IV software. Student's $t$ test was used to generate $P$ values, except for the experiments involving repeated measures, which were analyzed using 2-way ANOVA. Differences were considered significant if the probability of the difference occurring by chance was less than 5 in $100(P<0.05)$.

\section{Acknowledgments}

We thank Xiao-Ming Yin at Indiana University for providing Bid-KO mice, our surgeons and research nurses for helping collect the tissue samples, and Crissy Dudgeon and Brian Leibowitz for critical reading. This work is supported by NIH grants CA106348 and CA121105, American Cancer Society grant RSG-07-156-01-CNE, the Career Investigator Award from the American Lung Association/CHEST Foundation (to L. Zhang), the Flight Attendant Medical Research Institute (FAMRI), the Alliance for Cancer Gene Therapy (ACGT), American Cancer Society grant RGS-10-124-01-CCE, and NIH grants CA129829 and U01DK085570 (to J. Yu). L. Zhang is a scholar of the V Foundation for Cancer Research.

Received for publication October 11, 2010, and accepted in revised form January 26, 2011.

Address correspondence to: Lin Zhang, Hillman Cancer Center, the UPCI Research Pavilion, 5117 Centre Ave., Pittsburgh, Pennsylvania 15213, USA. Phone: 412.623.1009; Fax: 412.623.7778; E-mail: zhanglx@upmc.edu.

Bin Wu's present address is: Department of Gastroenterology, The Third Affiliated Hospital of Sun Yat-Sen University, Guangzhou, China. 
1. Xavier RJ, Podolsky DK. Unravelling the pathogenesis of inflammatory bowel disease. Nature. 2007;448(7152):427-434

2. Edelblum KL, Yan F, Yamaoka T, Polk DB. Regulation of apoptosis during homeostasis and disease in the intestinal epithelium. Inflamm Bowel Dis. 2006;12(5):413-424

3. Iwamoto M, Koji T, Makiyama K, Kobayashi N, Nakane PK. Apoptosis of crypt epithelial cells in ulcerative colitis. J Pathol. 1996;180(2):152-159.

4. Hagiwara C, Tanaka M, Kudo H. Increase in colorectal epithelial apoptotic cells in patients with ulcerative colitis ultimately requiring surgery. J Gas troenterol Hepatol. 2002;17(7):758-764.

5. Di Sabatino A, et al. Increased enterocyte apoptosis in inflamed areas of Crohn's disease. Dis Colon Rec tum. 2003;46(11):1498-1507.

6. Nenci A, et al. Epithelial NEMO links innate immunity to chronic intestinal inflammation. Nature. 2007;446(7135):557-561.

7. Eckmann L, et al. Opposing functions of IKKbeta during acute and chronic intestinal inflammation. Proc Natl Acad Sci US A. 2008;105(39):15058-15063.

8. Frey MR, Edelblum KL, Mullane MT, Liang D, Polk DB. The ErbB4 growth factor receptor is required for colon epithelial cell survival in the presence of TNF. Gastroenterology. 2009;136(1):217-226.

9. Schulzke JD, Bojarski C, Zeissig S, Heller F, Git ter AH, Fromm M. Disrupted barrier function through epithelial cell apoptosis. Ann N Y Acad Sci. 2006;1072:288-299.

10. Han X, et al. Regulation of intestinal barrier function by signal transducer and activator of transcription 5b. Gut. 2009;58(1):49-58.

11. Zeissig S, et al. Downregulation of epithelial apoptosis and barrier repair in active Crohn's disease by tumour necrosis factor alpha antibody treatment. Gut. 2004;53(9):1295-1302.

12. Marini M, et al. TNF-alpha neutralization ameliorates the severity of murine Crohn's-like ileitis by abrogation of intestinal epithelial cell apoptosis. Proc Natl Acad Sci U S A. 2003;100(14):8366-8371.
13. Yu J, Zhang L. PUMA, a potent killer with or without p53. Oncogene. 2008;27 suppl 1:S71-S83.

14. Wang P, Yu J, Zhang L. The nuclear function of p53 is required for PUMA-mediated apoptosis induced by DNA damage. Proc Natl Acad Sci U S A. 2007;104(10):4054-4059.

15. Ming L, Wang P, Bank A, Yu J, Zhang L. PUMA dissociates Bax and BCL-XL to induce apoptosis in colon cancer cells. J Biol Chem. 2006;281(23):16034-16042.

16. Yu J, Wang P, Ming L, Wood MA, Zhang L. SMAC/ Diablo mediates the proapoptotic function of PUMA by regulating PUMA-induced mitochondrial events. Oncogene. 2007;26(29):4189-4198.

17. Spehlmann ME, Eckmann L. Nuclear factor-kappa $\mathrm{B}$ in intestinal protection and destruction. Curr Opin Gastroenterol. 2009;25(2):92-99.

18. Wang $\mathrm{P}$, et al. PUMA is directly activated by NFkappaB and contributes to TNF-alpha-induced apoptosis. Cell Death Differ. 2009;16(9):1192-1202.

19. Qiu W, et al. PUMA regulates intestinal progenitor cell radiosensitivity and gastrointestinal syndrome. Cell Stem Cell. 2008;2(6):576-583.

20. Wu B, et al. p53 independent induction of PUMA mediates intestinal apoptosis in response to ischaemia-reperfusion. Gut. 2007;56(5):645-654

21. Cooper HS, Murthy SN, Shah RS, Sedergran DJ. Clinicopathologic study of dextran sulfate sodium experimental murine colitis. Lab Invest. 1993;69(2):238-249.

22. Siegmund B, Lehr HA, Fantuzzi G, Dinarello CA. IL-1 beta -converting enzyme (caspase-1) in intestinal inflammation. Proc Natl Acad Sci U S A. 2001;98(23):13249-13254.

23. Hibi T, Ogata H, Sakuraba A. Animal models of inflammatory bowel disease. J Gastroenterol. 2002;37(6):409-417.

24. Bauer A, et al. The NF-kappaB regulator Bcl-3 and the BH3-only proteins Bim and Puma control the death of activated T cells. Proc Natl Acad Sci U S A. 2006;103(29):10979-10984.

25. Ina K, et al. Resistance of Crohn's disease T cells to multiple apoptotic signals is associated with a Bcl-2/Bax mucosal imbalance. J Immunol. 1999;163(2):1081-1090.

26. Peppelenbosch MP, van Deventer SJ. T cell apoptosis and inflammatory bowel disease. Gut. 2004;53(11):1556-1558.

27. Adams JM, Cory S. The Bcl-2 apoptotic switch in cancer development and therapy. Oncogene. 2007;26(9):1324-1337.

28. Wang J, Fu YX. Tumor necrosis factor family members and inflammatory bowel disease. Immunol Rev. 2005;204:144-155.

29. Gao J, et al. IRF-1 transcriptionally upregulates PUMA, which mediates the mitochondrial apoptotic pathway in IRF-1-induced apoptosis in cancer cells. Cell Death Differ. 2010;17(4):699-709.

30. Yu J, Zhang L. The transcriptional targets of p53 in apoptosis control. Biochem Biophys Res Commun. 2005;331(3):851-858

31. Hofseth LJ, et al. Nitric oxide-induced cellular stress and p53 activation in chronic inflammation. Proc Natl Acad Sci U S A. 2003;100(1):143-148.

32. You $\mathrm{H}$, et al. FOXO3a-dependent regulation of Puma in response to cytokine/growth factor withdrawal. J Exp Med. 2006;203(7):1657-1663.

33. Podolsky DK. Inflammatory bowel disease. NEngl JMed. 2002;347(6):417-429.

34. Qiu W, Leibowitz B, Zhang L, Yu J. Growth factors protect intestinal stem cells from radiation-induced apoptosis by suppressing PUMA through the PI3K/ AKT/p53 axis. Oncogene. 2010;29(11):1622-1632.

35. Yin XM, et al. Bid-deficient mice are resistant to Fas-induced hepatocellular apoptosis. Nature. 1999;400(6747):886-891.

36. Wirtz S, Neufert C, Weigmann B, Neurath MF. Chemically induced mouse models of intestinal inflammation. Nat Protoc. 2007;2(3):541-546.

37. Qiu W, Carson-Walter EB, Kuan SF, Zhang L, Yu J. PUMA suppresses intestinal tumorigenesis in mice. Cancer Res. 2009;69(12):4999-5006.

38. Maeda S, et al. Nod2 mutation in Crohn's disease potentiates NF-kappaB activity and IL-1beta processing. Science. 2005;307(5710):734-738. 